\title{
COVID-19 virtual clinical skills learning: virtual emotive patient avatar with augmented reality sign
}

\author{
Arkendu Sen, A., Lim, Z.Y., Tha, K.K., Selvaratnam, L. \\ Monash University, Malaysia
}

\section{Background:}

Clinical skills practice has increasingly been introduced in the early years for medical and health sciences curricula through patient encounters involving paid "simulated patients (SPs)". Normal SPs offer a low level of fidelity, and the level of authenticity is a challenge as students do not always get to experience the range of patient signs and problems. Such clinical signs, to be realistic and authentic, in a virtual environment in COVID 19 situation could be created in augmented reality (AR) and integrated with the virtual patient.

\section{Method:}

The present authors who have recently developed discrete AR clinical signs as a pilot project intended to extend this to virtual emotive patients' avatars (VEPA) to give students authentic learning experience of a real-life patient. Some similar work is scantly available that incorporates emotional expression normally displayed by real patients in a real clinical setting. The implementation of such concepts is challenging as there are no established freely available projects of VEPA inclusive of an AR clinical signs overlay. With various versions of VEPA for history taking skills and for physical examination of simulated clinical signs (with AR overlays), students will be able to experience real-life virtual SPs through these VEPA individually as well as during student discussions and discourses in a shared virtual space to help in their learning and practice/review of clinical skills.

\section{Conclusion:}

VEPA eliminates the risk of harm to the actual patients, especially in acute cases, and is non-invasive and noncontagious in COVID 19 situations avoiding any physical contact. VEPA engages learners in a shared virtual space. In areas of Medicine and Health Sciences, VEPA will benefit through a generic Virtual avatar for authentic Industry experiences in areas of a) Medical patient examination b) Pharmacy medicine dispensing and counselling c) Professional counselling. VEPA will be useful especially in COVID and post COVID authentic near real-life hospital experience in the current Industry 4.0 transformation.

Keywords: Augmented Reality, Avatar, Simulation Skills.

16 